\title{
Experience of ReCell in Skin Cancer Reconstruction
}

\author{
Onur Gilleard, Nicholas Segaren, Ciaran Healy \\ Department of Plastic Surgery, St Thomas Hospital, London, UK
}

The ReCell system (Avita Medical) is a cell culture product that allows the immediate processing of a small split-thickness skin biopsy to produce a complete population of cells including keratinocytes, melanocytes, Langerhans cells and fibroblasts. This series is the first to highlight the reconstructive applications of ReCell following ablative skin cancer surgery. The ReCell system was utilized for three patients following skin cancer excision. In two cases, the cells were applied to forehead flap donor sites following nasal reconstruction. In one case, the cells were applied to the calvarial periosteum following wide local excision of a melanoma scar. Assessment of the treated area was performed using the patient and observer scar assessment scale after 1 year. The Patient and Observer Scar Assessment Scale (POSAS) scores for the 2 patients treated with ReCell following forehead flap surgery were 22 and 32 . The score for the patient that underwent wide local excision of a melanoma scar was 45 . The absence of a donor site, accelerated healing and the satisfactory aesthetic appearance of the mature scars in this series suggest that ReCell may play a useful role in reconstruction following skin cancer excision.

Keywords Guided tissue regeneration / Wound closure techniques / Skin
Correspondence: Onur Gilleard Department of Plastic Surgery, St Thomas Hospital, 53 Treadwell Rd, Epsom, Surrey, KT18 5JR, London, UK Tel: +44-1372722695

Fax: +44-1372724367

E-mail: onurgilleard@aol.com.

Received: 20 Mar 2013 • Revised: 10 May 2013 - Accepted: 24 May 2013

pISSN: 2234-6163 • elSSN: 2234-6171 • http://dx.doi.org/10.5999/aps.2013.40.5.627 • Arch Plast Surg 2013;40:627-629

No potential conflict of interest relevant to this article was reported.

\section{INTRODUCTION}

Traditional methods to reconstruct defects following skin cancer excision include skin grafting, loco-regional flaps and free flaps. In this series we report the use of a new skin cell culture system that results in insignificant donor site morbidity, as an alternative or adjunct to these techniques.

The ReCell system (Avita Medical, Cambridge, UK) is a cell culture product that allows the immediate processing of a small split-thickness skin biopsy to produce a complete population of cells including keratinocytes, melanocytes, Langerhans cells and fibroblasts. It was introduced into clinical practice in 2005 and has so far been used primarily in the treatment of partial thickness burns. Within this context, ReCell has been shown to promote healing and results in scars of comparable quality to skin grafting $[1]$.

This series is the first to highlight the reconstructive applications of ReCell following ablative skin cancer surgery.

\section{CASE}

\section{Using the ReCell system}

In accordance with the manufacturer guidelines, the following steps were undertaken: a $10 \mathrm{~mm} \times 5 \mathrm{~mm}$ strip of split thickness skin graft was harvested from a region adjacent to the wound requiring reconstruction, using a scalpel blade. The skin was put in $5 \mathrm{~mL}$ of trypsin solution for 10 minutes at $37^{\circ} \mathrm{C}$ to facilitate intercellular detachment. During this time, the scalpel blade 
was used to continually scrape off the epidermal cells from the dermis. The cells were then transferred to a lactate solution. The resulting suspension was aspirated with a $5-\mathrm{mL}$ syringe and sprayed evenly onto the forehead flap donor site ( 2 cases) or calvarial periosteum ( 1 case). A non-absorbent dressing Telfa clear (Covidien, Dublin, Ireland) was applied and left in place for 1 week. A dry gauze dressing was used for an additional 2 weeks, after which the wound was left open and required no further treatment.

\section{ReCell on forehead flap donor sites Case 1}

A 39-year-old man underwent Moh's micrographic surgery to treat an infiltrative basal cell carcinoma (BCC) arising from the right nasal sidewall. The resulting full thickness defect measured $2 \mathrm{~cm} \times 2 \mathrm{~cm}$ and involved the dorsum of the nose, the right side wall, and the nasal tip. A three-stage forehead flap was planned for reconstruction.

During the first stage of surgery, in order to improve the final aesthetic outcome, the remainder of the involved subunits and the left nasal side wall were completely excised. The resulting final defect measured $4 \mathrm{~cm} \times 5 \mathrm{~cm}$. Reconstruction of the lining and structural support of the nose were achieved using a mucosal advancement flap and septal cartilage grafts, respectively. A template using the excised nasal skin was used to accurately mark out a paramedian forehead flap. The flap was raised from its superior aspect to incorporate the skin, subcutaneous fat and part of the frontalis muscle. It was rotated 180 degrees and inset without tension. Once the primary nasal reconstruction was complete, the inferior aspect of the donor site defect was closed directly.

The superior aspect of the donor site measured $6 \mathrm{~cm} \times 5 \mathrm{~cm}$ (Fig. 1A) and was treated using the ReCell system in the manner described above.

\section{Fig. 1. The forehead flap donor site}

ReCell was used to treat the forehead flap donor site. (A) The donor site immediately after the flap was raised. (B) The donor site 1 year after treatment with ReCell.
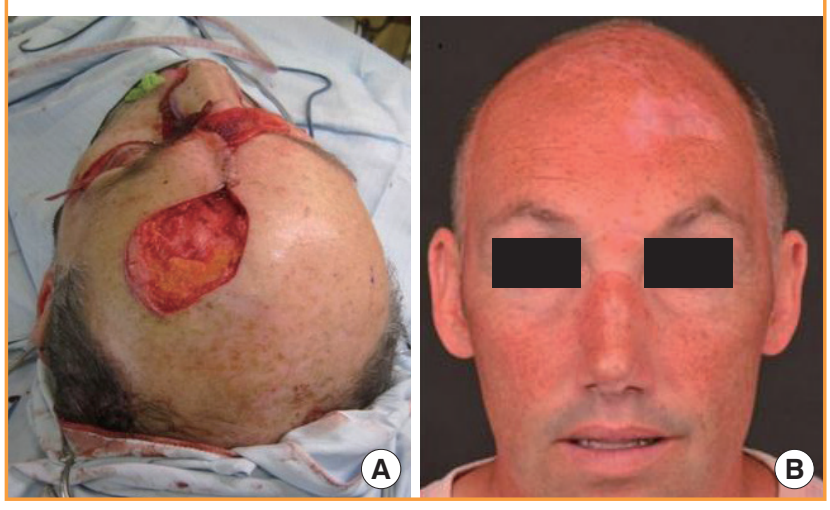

Photographic assessment of the donor site was undertaken at 1 year (Fig. 1B) [2]. The Patient and Observer Scar Assessment Scale (POSAS) [2] yielded a score of 32 (11 = normal skin, 110 worst scar imaginable).

\section{Case 2}

A 72-year-old man with a medical history of type 2 diabetes underwent Moh's micrographic surgery to treat a morphoeic BCC arising from the nasal tip. The resulting full-thickness defect measured $3 \mathrm{~cm} \times 2 \mathrm{~cm}$ and involved the nasal tip, the dorsum of the nose and both side walls. A three-stage forehead flap was planned for reconstruction and was performed in a manner similar to that described for case 1 .

Following the first stage of surgery, the donor site was initially allowed to heal by secondary intention. One month later, it was noted that healing was delayed and epithelialization was minimal (Fig. 2A). Consequently, during the second stage of surgery, undertaken 6 weeks after the primary reconstructive procedure, the donor site was treated using the ReCell system in the manner described above.

Photographic assessment of the donor site was performed 1 year after the application of ReCell (Fig. 2B). The POSAS score was 22.

\section{ReCell to reconstruct a scalp wound following WLE of malignant melanoma}

\section{Case 3}

A 42-year-old female patient underwent a 2-cm wide local excision (WLE) of a malignant melanoma scar on her scalp (Fig. $3 \mathrm{~A})$. The resulting wound measured $5 \mathrm{~cm} \times 7 \mathrm{~cm}$ with periosteum forming the base (Fig. 3B). The patient was concerned about the aesthetic problem of alopecia following a skin graft as well as donor site morbidity. As a result, the ReCell system was

Fig. 2. The forehead flap donor site during the second stage of surgery

ReCell was used to treat the forehead flap donor site during the second stage of surgery. (A) Forehead flap donor site 1 month after the flap was raised. (B) The donor site 1 year after treatment with ReCell.
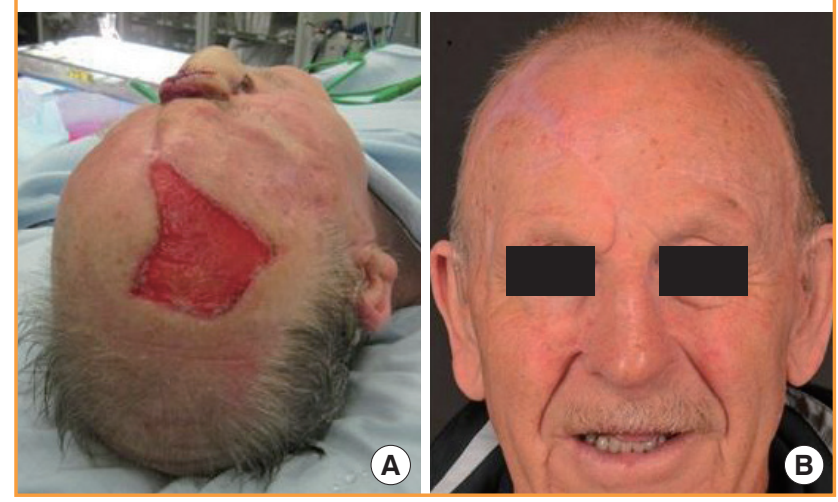


\section{Fig. 3. Scalp defect after WLE of melanoma}

ReCell was used to reconstruct the defect following 2-cm WLE of a melanoma scar. (A) 2-cm WLE marked. (B) Scalp defect following melanoma scar excision with periosteum at the base. (C) Scar 1 year after treatment with ReCell. WLE, wide local excision.
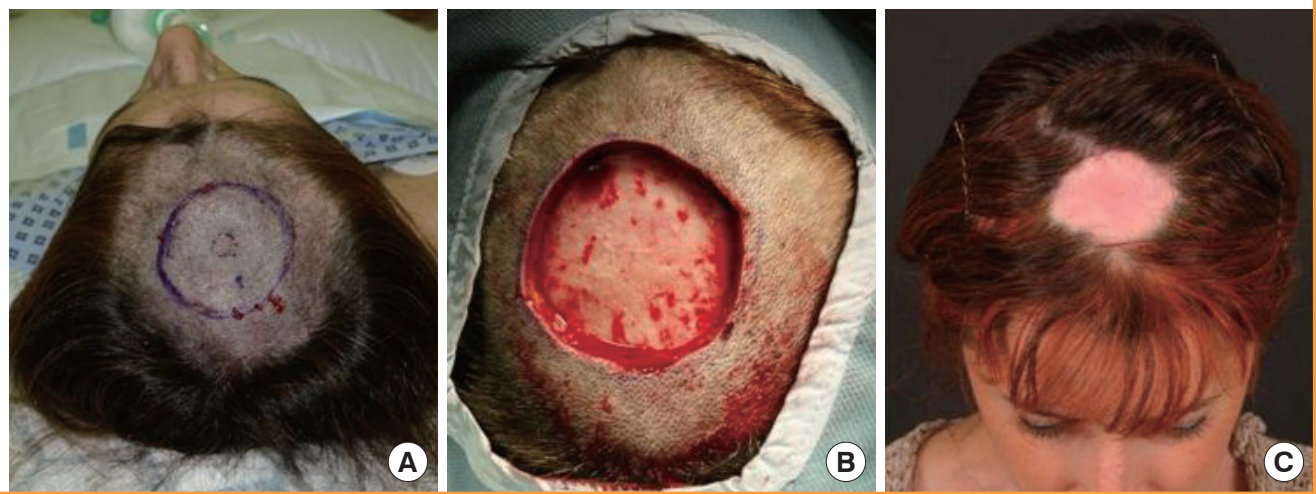

used in the manner described above. At 1 year, the wound had contracted sufficiently to be covered by the patient's hair, making it imperceptible during day-to-day activities (Fig. 3C). The POSAS score was 45.

\section{DISCUSSION}

Keratinocyte cultures have been used in the treatment of burns for over 20 years [3]. The disadvantages of this treatment modality include the need to have a laboratory close to the clinical area in order to culture the cells and the relatively poor aesthetic restoration of skin due to the absence of certain epidermal elements, such as melanocytes, amongst the cultured cells [4]. The ReCell system, which allows immediate processing of skin in the operating theatre to produce a complete population of cells, was introduced into clinical practice in 2005. It has so far been used primarily in the treatment of partial thickness burns. Within this context, this autologous cell harvesting device has been shown to promote healing and result in scars of comparable quality to skin grafting [1].

The senior author has been using the 3-stage forehead flap to reconstruct extensive nasal defects following skin cancer ablation for 10 years. Although the final results of nasal reconstruction using this technique are usually excellent, the management of large donor site defects remains difficult [5]. Skin grafting the area results in a clearly delineated scar, which is of different pigmentation and texture to the surrounding skin. In addition, a significant further donor site is created. Allowing the wound to heal by secondary intention usually results in a better final aesthetic outcome than skin grafting but has the disadvantage of a longer healing process, requiring a protracted course of dressings. The forehead flap donor site is a full thickness wound with no residual dermal component to spray the processed epidermal cells onto. Despite this, using ReCell has accelerated the healing process whilst negating the need to produce a significant further donor site. Dressings were only required for 3 weeks after surgery, and the scars in the two cases were deemed highly satisfactory by both the clinician and patient.

In the reconstruction of the periosteal scalp defect following wide local excision of melanoma, the use of ReCell allowed significant wound contracture, which enabled the area of alopecia to be covered by the patient's hair. The wound itself required dressings for a period of 3 weeks. Treatment of the wound using ReCell in this case allowed healing in a similar time frame to skin grafting without creating a donor site, whilst also resulting in wound contracture to a degree similar to that seen following healing by secondary intention.

The absence of a donor site, accelerated healing and the satisfactory aesthetic appearance of the mature scars in this series together suggest that ReCell may have a useful role to play in reconstruction following skin cancer excision.

\section{REFERENCES}

1. Gravante G, Di Fede MC, Araco A, et al. A randomized trial comparing ReCell system of epidermal cells delivery versus classic skin grafts for the treatment of deep partial thickness burns. Burns 2007;33:966-72.

2. Draaijers LJ, Tempelman FR, Botman YA, et al. The patient and observer scar assessment scale: a reliable and feasible tool for scar evaluation. Plast Reconstr Surg 2004;113:1960-5.

3. Chester DL, Balderson DS, Papini RP. A review of keratinocyte delivery to the wound bed. J Burn Care Rehabil 2004;25:266-75.

4. Green H, Kehinde O, Thomas J. Growth of cultured human epidermal cells into multiple epithelia suitable for grafting. Proc Natl Acad Sci U S A 1979;76:5665-8.

5. Menick FJ. A 10-year experience in nasal reconstruction with the three-stage forehead flap. Plast Reconstr Surg 2002;109:1839-55. 